\title{
Chinese Herbal Medicine in Treating Primary Sjögren's Syndrome: A Systematic Review of Randomized Trials
}

\author{
Hui Luo, ${ }^{1}$ Xinxue Li, ${ }^{1}$ Jianping Liu, ${ }^{1}$ Flower Andrew, ${ }^{2}$ and Lewith George ${ }^{2}$ \\ ${ }^{1}$ Center for Evidence-Based Chinese Medicine, Beijing University of Chinese Medicine, Chaoyang District, Beijing 100029, China \\ ${ }^{2}$ Center for Research on Complementary and Alternative Medicine, University of Southampton, Primary Care, \\ Southampton SO16 5ST, UK
}

Correspondence should be addressed to Jianping Liu, jianping_l@hotmail.com

Received 20 February 2012; Revised 4 May 2012; Accepted 8 May 2012

Academic Editor: Weidenhammer Wolfgang

Copyright (c) 2012 Hui Luo et al. This is an open access article distributed under the Creative Commons Attribution License, which permits unrestricted use, distribution, and reproduction in any medium, provided the original work is properly cited.

\begin{abstract}
Background. There is no curative treatment for primary Sjögren's syndrome (PSS). Chinese herbal medicine (CHM) is widely used in the treatment of PSS in China. Objective. To evaluate the effectiveness and safety of CHM for PSS. Methods. PubMed, Cochrane Library, China Knowledge Resource Integrated Database, Chinese Biomedical Database, Wanfang Data, and the Database for Chinese Technical Periodicals were searched for randomized controlled trials (RCTs) of CHM or CHM plus conventional medicine for PSS compared with placebo or conventional medicine. RevMan 5.0.17 was employed to conduct data analyses and assess homogeneity. Statistical models were chosen according to heterogeneity. Results. A total of 52 RCTs were included. The overall methodological quality of included trials was low. 49 trials reported response rates, of which 32 found significant improvements favoring CHM treatment against controls; 20 trials reported lacrimal function by Schirmer test scores, of which 16 trials reported a significant difference favoring CHM treatment. 21 trials reported salivary function by salivary flow rate, of which 10 reported significant favorable effects of CHM treatment. Other trials found no difference. The reported adverse effects of CHM included nausea, diarrhea, and other minor digestive symptoms, but more frequent adverse effects occurred in conventional medicine groups. Conclusions. Preliminary evidence from RCTs suggests the effect of CHM is promising for relieving symptoms, improving lacrimal and salivary function in PSS. However, the poor methodological quality of the included trials means that further welldesigned, multicentered, larger trials are needed.
\end{abstract}

\section{Introduction}

Sjögren's syndrome, also known as "Mikulicz's disease" and "Sicca syndrome," is a diffuse connective tissue disease in which immune cells attack and destroy the exocrine glands that produce tears and saliva [1]. Clinical symptoms of the disorder typically involve dryness of the mouth and eyes [2]. In addition, Sjögren's syndrome may affect other exocrine glands and organs of the body and cause multisystem signs and symptoms such as fatigue and joint pain. If a patient manifests the above symptoms together with positive blood tests for inflammatory and autoimmune markers, and other connective tissue diseases are eliminated, it suggests a diagnosis of primary Sjögren's syndrome (PSS) [3]. Further diagnostic confirmation can be obtained by the Schirmer test that measures tear production and biopsy of the salivary glands. Primary Sjögren's syndrome has developed into a global disease. It is estimated to affect as many as 3 million people in the USA alone making it one of the most common autoimmune rheumatic diseases [4]. In Europe, the prevalence of Sjögren's syndrome in the UK is estimated at approximately $0.33 \%$ [5], Greece $0.09 \%$ [6], and Slovenia $0.6 \%$ [ 7 ]. In China, the prevalence is $0.3 \%$. Sjögren's syndrome incidence rates peak at age 45 to 50 , and nine out of ten patients are women. Sometimes, it occurs in children $[1,8]$.

There are currently no known curative treatments for Sjögren's syndrome. Management is symptomatic and the most common treatments are prednisone, immunosuppressant, and symptomatic support, with the aim of relieving clinical symptoms and preventing organs being damaged by disease progression. Moisture replacement therapies such 
as artificial tears can relieve the symptoms (dry eyes) and corticosteroids or immunosuppressive drugs may be prescribed to control severe complications $[1,9]$. These treatments are based on the experience of clinical physicians, expert opinion, and very limited clinical research. In 2010, a systematic review of treatment for PSS published in JAMA indicated the very limited evidence available for commonly used drugs and there is almost no evidence for any treatment for patients who do not respond to first-line therapies. We need more research for this relatively common and disabling condition if we are to provide effective, evidence-based, interventions [10].

There is no record of Sjögren's syndrome as such in the classical literature of traditional Chinese medicine (TCM), but knowledge of its clinical manifestations and symptomatic treatment can be traced back over 2000 years. Modern TCM researchers have conducted clinical trials on Sjögren's syndrome. A systematic review of these trials from 1997 to 2010 analyzed and evaluated randomized controlled trials of the Chinese herbal medicine (CHM) of treatment of Sjögren's syndrome [11]. However, the systematic review was written in Chinese and published in a Chinese journal and trials that recruited both primary and secondary Sjögren's syndrome patients or patients with severe complications were included in the paper. The methodological quality of the included trials at this point in time was generally poor, but over the past year 10 new RCTs have been published with a substantially improved methodological quality. Therefore, the objective of this systematic review is to appraise existing RCTs on CHM for PSS and provide an up-to-date evidencebased evaluation on the effectiveness and safety of CHM for PSS.

\section{Methods}

2.1. Eligibility Criteria. RCTs of CHM treating PSS were included, with no limitations on language or publication format. Trials were eligible when the study participants were patients with PSS, and there were no limitations on the participant's age, gender, and nationality. Interventions included any form of CHM (including prescribed formulae, patent medicines, herbal decoctions, herbal extracts and herbal injections) based on syndrome differentiation, and treatment, used as a sole treatment or in conjunction with conventional therapies. Control treatments included standard conventional treatment, placebo, and waiting list controls. Outcomes included total effectiveness rate (response rate), lacrimal function, salivary gland function, quality of life, and adverse events.

Trials were excluded if any of the following were identified: (1) The study population included patients with secondary Sjögren's syndrome, since this could not be differentiated from PSS; (2) if they involved the treatment of complications of PSS such as severe hepatic, renal damage or hematological damage; (3) if information about the participants or intervention was not clearly reported; (4) if controlled treatment included any use of CHMs as, in this case, it would be impossible to evaluate the specific effects of the intervention.
2.2. Information Sources. Both Chinese databases and English databases were searched, including China Knowledge Resource Integrated Database (CNKI), the Database for Chinese Technical Periodicals (VIP), Chinese Biomedical Database (CBM), Wanfang Data, PubMed, and the Cochrane Library (2012, Issue 2).

2.3. Searching. In the Chinese databases, we employed Sjögren's syndrome and random* as the main search terms without limitation on the modalities CHM employed. We searched PubMed by using the MeSH term Sjögren's syndrome with the following restrictions: humans as study participants, RCTs, or meta-analysis. When searching the Cochrane Library, we used Sjögren's syndrome as the key search word. We searched all articles on treatment for PSS published before February 10, 2012. The search strategy for the databases was provided in Table 3.

2.4. Selection. We employed the trial selection methods described in the Cochrane Handbook for Systematic Reviews of Interventions, version (1) import the search results from different databases into the reference management software NoteExpress2 (2.6 version); (2) exclude irrelevant articles by reading titles and abstracts; (3) obtain the full papers for all possibly relevant trials; (4) exclude articles with duplicate publication; (5) contact the authors when the data was not available; (6) recheck identified articles according to the above steps; (7) include the final trials for the review [68].

2.5. Data Collection Process. Data from the included trials were extracted by two authors independently. Any discrepancies were resolved by referral to the original article and, if necessary, a third author was consulted. The following data were extracted from included trials: methodological components, participant characteristics, interventions and controlled treatments, and outcome measures.

2.6. Risk of Bias in Individual Studies. The Cochrane Collaboration's tool for assessing the risk of bias was used to evaluate the methodological quality of included trials [68], which covers six domains of bias: selection bias, performance bias, detection bias, attrition bias, reporting bias, and other bias. Judgments were reached according to the text description or summary of relevant trial characteristics for each item in the tool.

2.7. Summary Measures and Synthesis of Results. Dichotomous data (response rate) was presented as risk ratio (RR) and continuous data outcomes (including lacrimal function, salivary gland function, and quality of life) as mean difference (MD), both with $95 \%$ confidence intervals (CI). RevMan 5.0.17 was employed to conduct data synthesis. Homogeneity of risk ratio or mean difference in trials with unequal sample sizes within one type of comparisons was analyzed using $\mathrm{I}^{2}$ and $\mathrm{Z}$ values, and statistical models were chosen based on significance of heterogeneity. 


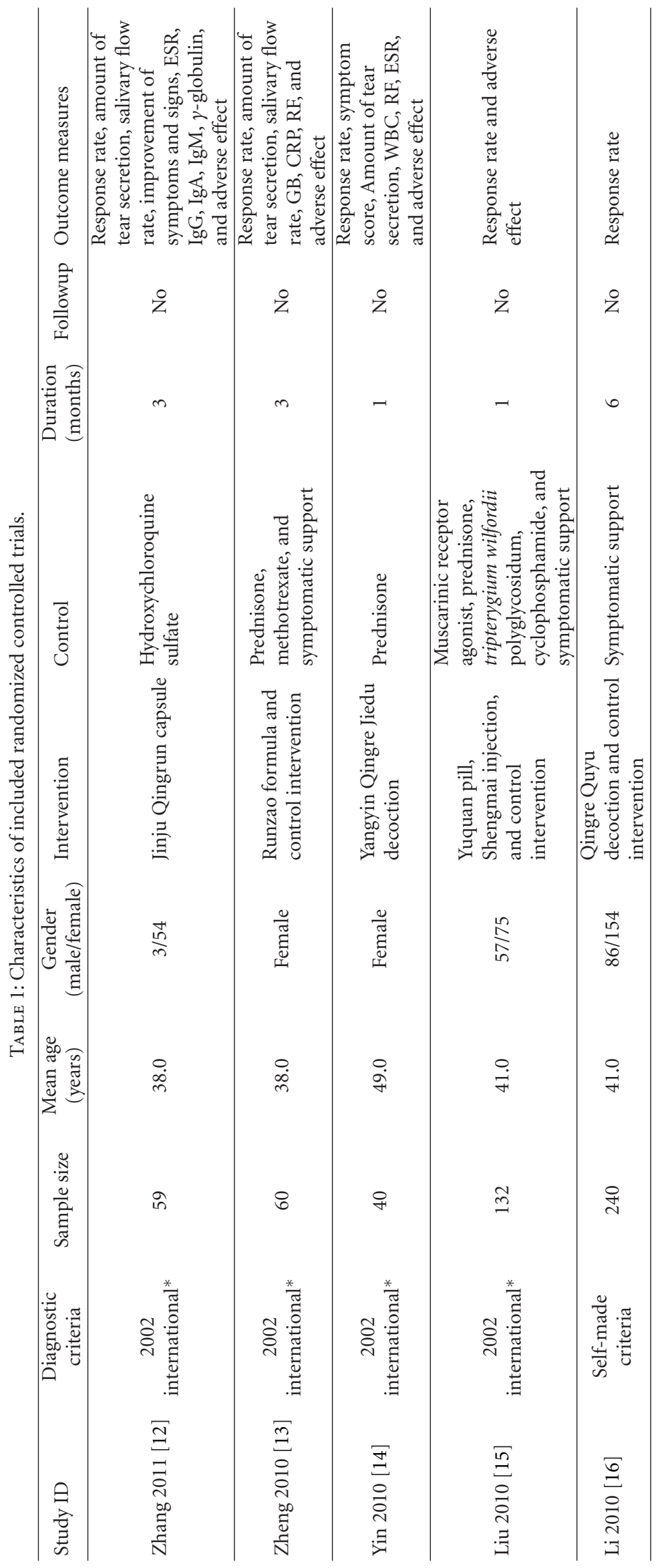




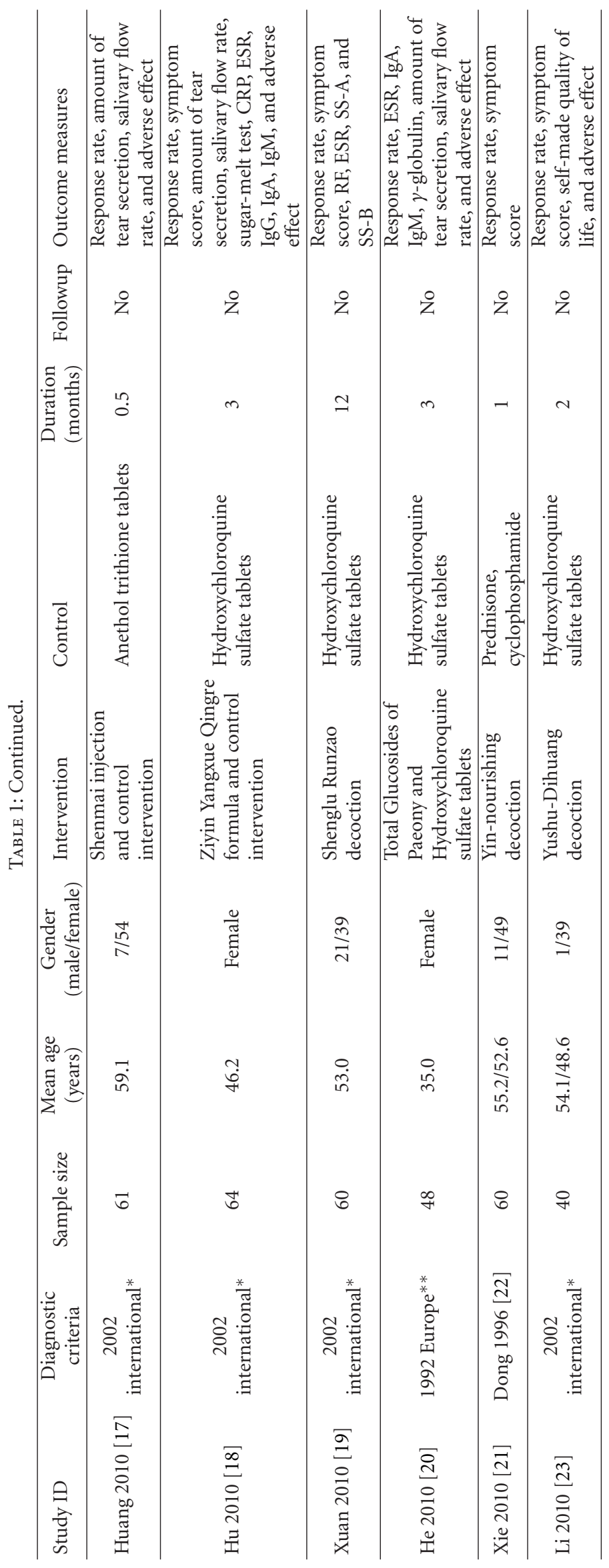




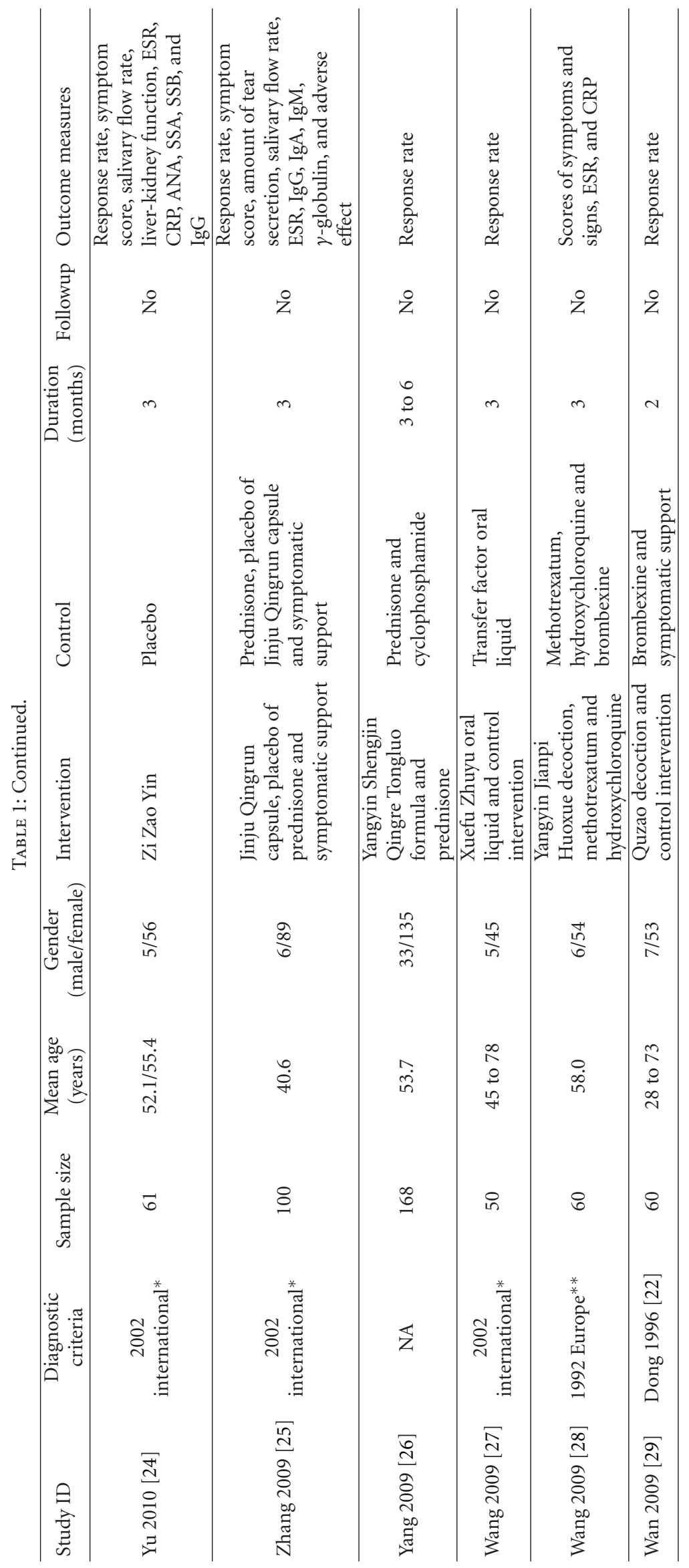




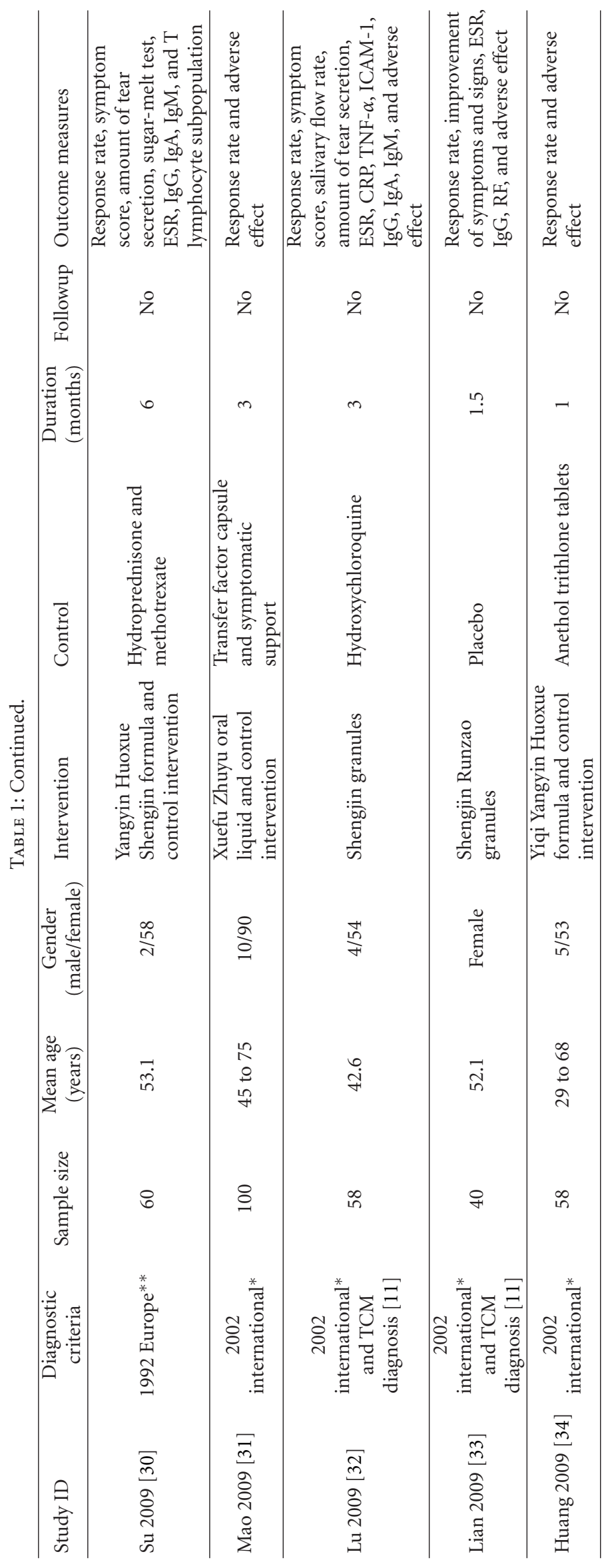




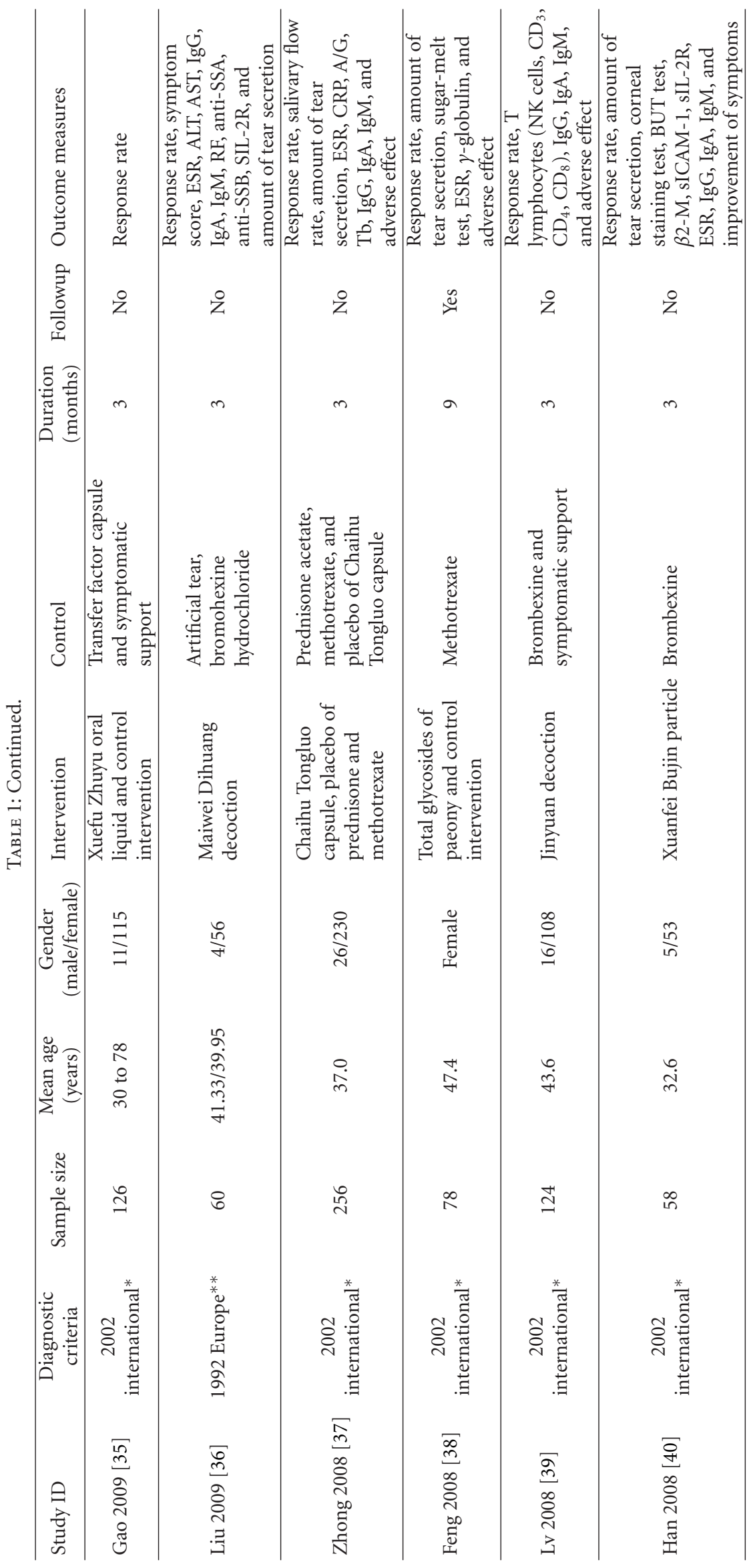




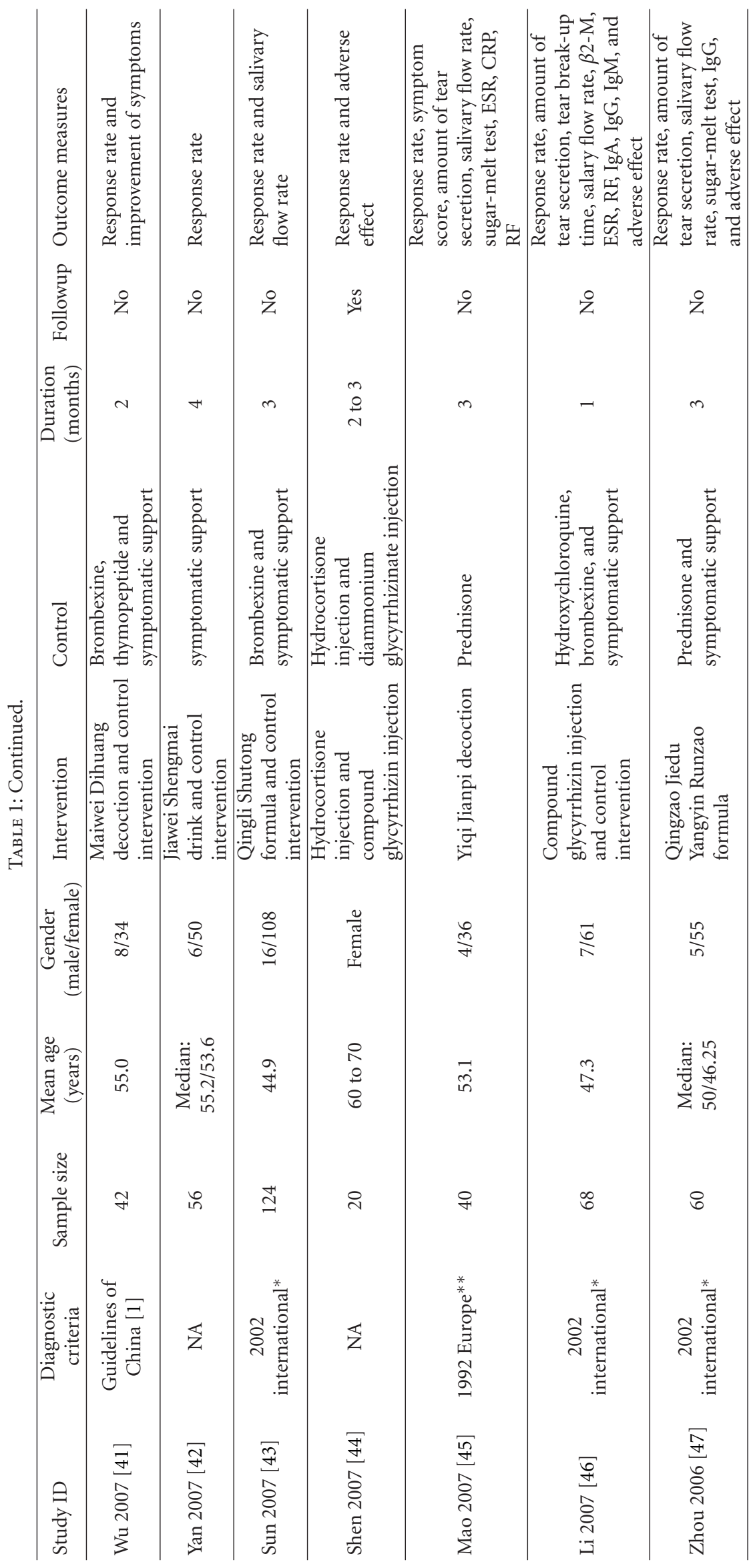




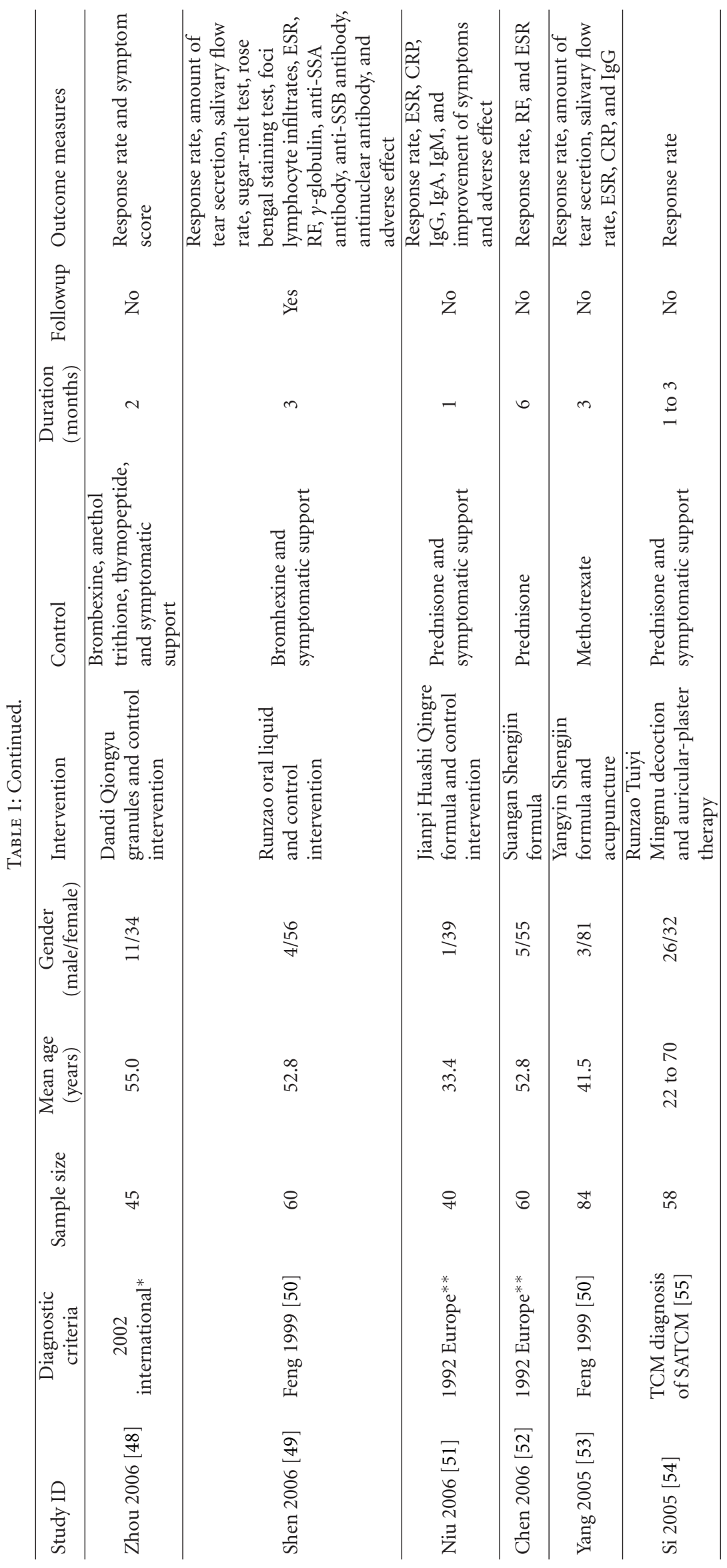




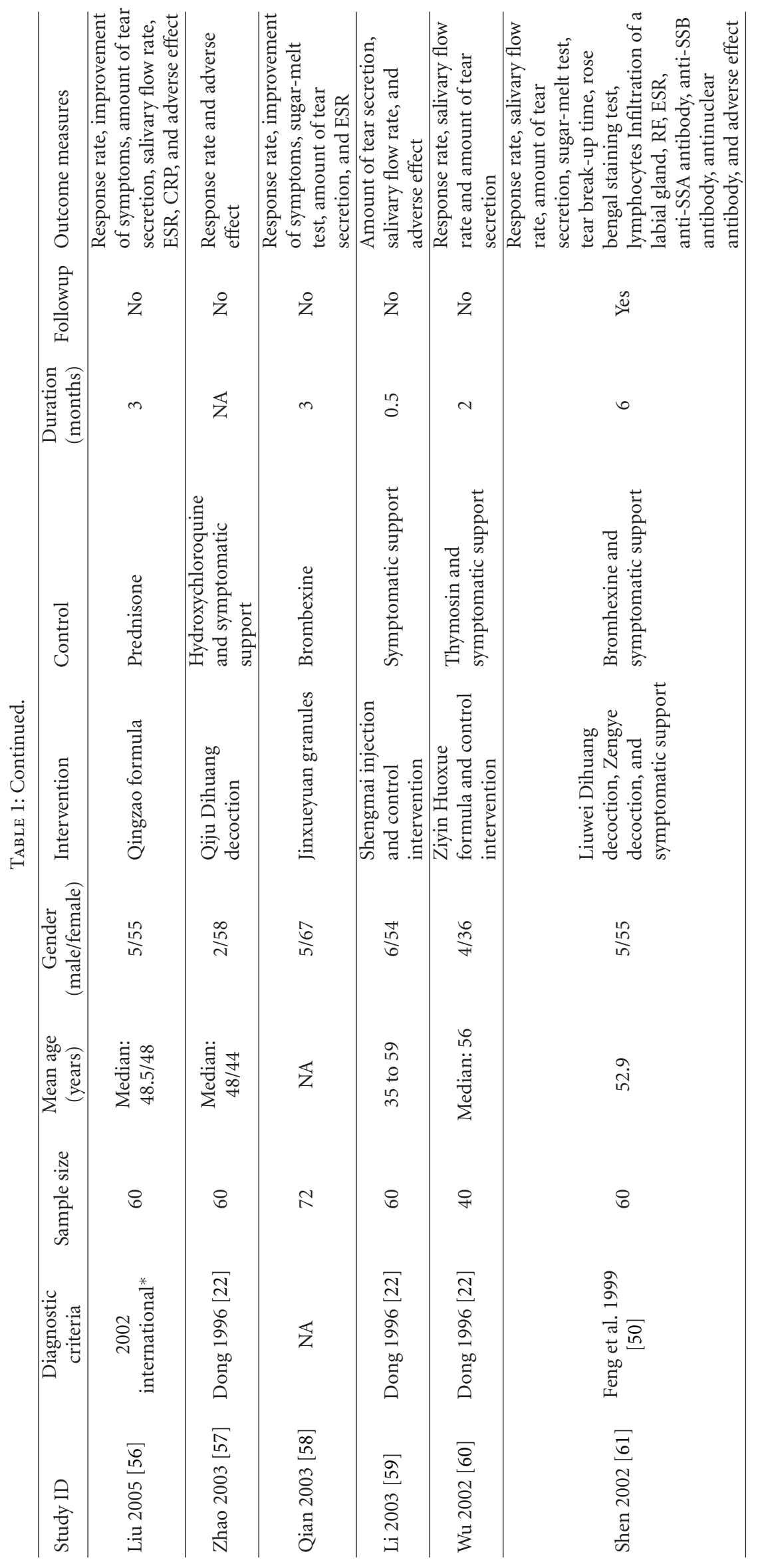




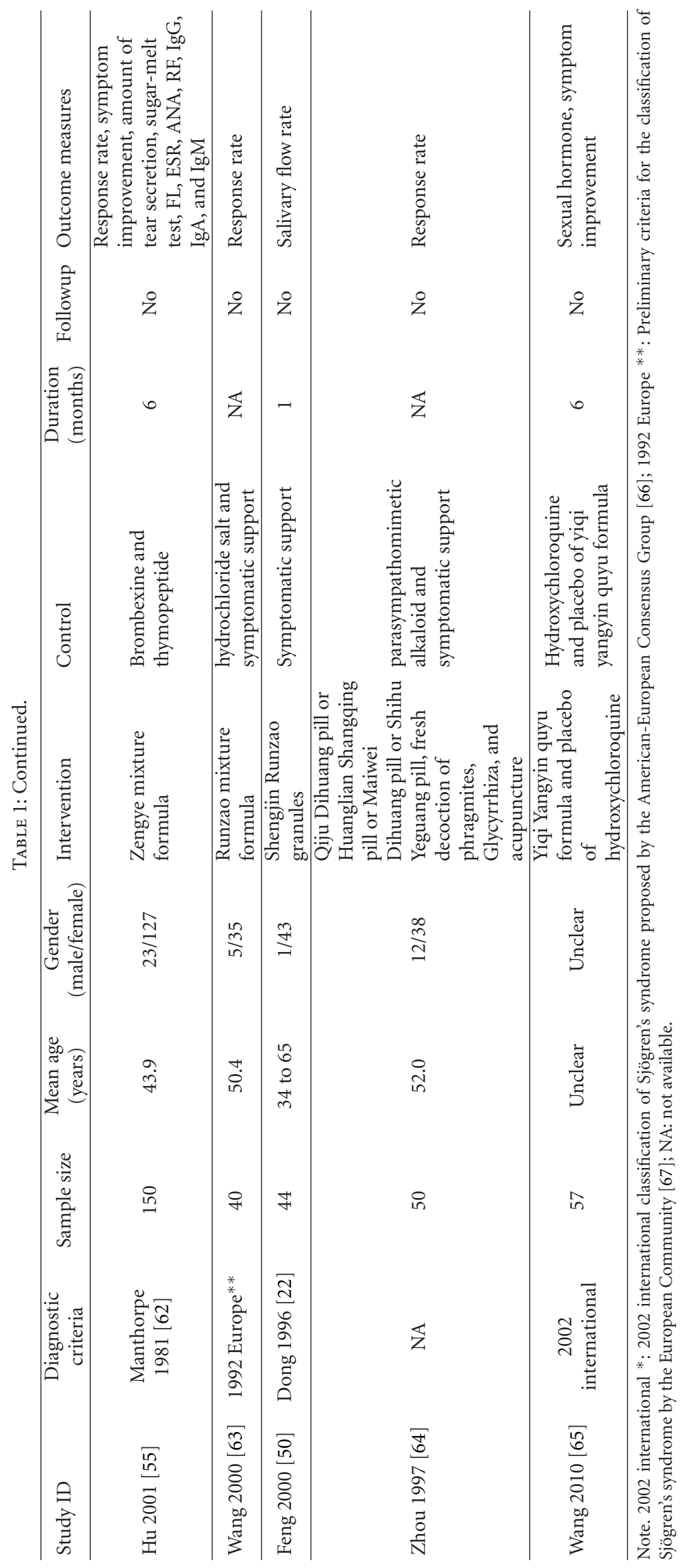




\section{Results}

3.1. Study Selection. A total of 559 articles were found from the initial searches. After reading titles and abstracts, 60 full-text papers were retrieved and further 8 studies were excluded for the following reasons: 2 trials were excluded for involving patients with haematological complications [69] and kidney damage [70]; 3 trials were excluded due to CHM treatment in control group [71-73]; 1 trial was excluded due to being nonrandomized trial [74]; 2 trials were excluded due to duplication $[75,76]$. Finally, 52 RCTs, which were published in Chinese, were judged to be eligible and included in the review [12-21, 23-61, 63-65] (Figure 1).

3.2. Study Characteristics. All the included trials were conducted in China, with a total number of 3,829 PSS patients, $12.9 \%$ of them were male $(n=495)$ and $85.5 \%$ were female ( $n=3,272)$, and data on gender were not available in two trials $(1.6 \%)$. The sample size of the included trials ranged from 20 to 256 participants with an average of 74 patients per trial. No trials reported how they estimated the sample size. The diagnostic criteria of PSS included the 2002 international classification of Sjögren's syndrome [66], the 1992 European diagnostic criteria [67], and other foreign or Chinese established criteria referred to in previous publications $[1,22,62,77,78]$.

CHM therapy followed traditional treatment principles to nourish yin, moisten dryness and generate body fluids, replenish qi and blood, tonify the "lungs" and "spleen," strengthen the "spleen" to remove dampness, activate blood to remove stasis, and clear fire poison. CHM treatment principles also included dispersing and reinforcing methods to treat diseases caused by excess and deficiency patterns. This reflects the features of a very mixed syndrome presentations in PSS patients. The herbal medicines included multicomponent decoctions, patent medicines (pills and tablets), concentrated herbal granules, and herbal injections. Twenty trials tested patent medicines and fixed formula granules, while 32 trials tested individualized herbal decoctions, permitting modified formulae according to individual participant's TCM syndrome. Conventional treatments included (1) symptomatic management, such as artificial tears, oculenta, artificial saliva, bromhexine, ambroxol hydrochloride, and parasympathomimetic alkaloid; (2) corticosteroids, such as prednisone; (3) immunosuppressive drugs, such as methotrexate, hydroxychloroquine, and cyclophosphamide; (4) other treatment, such as thymosin, vitamins, antibiotics, transfer factor, and nonsteroidal anti-inflammatory drugs.

All the included trials were classified into four comparisons according to the interventions: (1) CHM versus placebo $(n=2)$; (2) CHM versus conventional medicine ( $n=19$ ), in which 2 trials compared CHM plus placebo of conventional medicine to conventional medicine plus placebo of CHM [37, 65]; (3) CHM plus conventional medicine versus conventional medicine $(n=28)$, in which one trial compared CHM plus conventional medicine to conventional medicine plus placebo of CHM [25]; (4) CHM plus acupuncture versus conventional medicine $(n=3)$.
48 different CHMs were tested in 52 trials. Two patent medicines and one herbal injection, Jinju Qingrun capsule [12, 25], Xuefu Zhuyu oral liquid [27, 31, 35, 44, 46] and Glycyrrhizin Compound injections [44, 46], were studied in three and two trials, respectively. Due to the significant heterogeneity of the interventions, only two trials on Jinju Qingrun capsule could be pooled in a meta-analysis [12, 25].

Outcome measurements included response rate (total effectiveness rate), salivary gland function, lacrimal function, laboratory findings, TCM syndrome evaluation scores, and adverse events.

The response rate was a composite outcome index used throughout these trials, integrating factors from symptoms, signs, and laboratory findings [79]. The effect was considered positive when (1) clinical symptoms of dry mouth and eyes were markedly improved; (2) the value of Schirmer test and salivary flow rate were markedly increased. If there was no change evident in clinical symptoms or lab testing, then it was considered to be ineffective.

Salivary gland function was tested by collecting saliva and determining the amount produced in a five-minute period, or by sugar-melt test. Lacrimal function was evaluated by Schirmer test to measure the production of tears. Laboratory findings of included trials included erythrocyte sedimentation rate (ESR), C-reactive protein (CRP), rheumatoid factor (RF), antinuclear antibody (ANA), anti-Sjögren's syndrome A antibody (anti-SSA), anti-Sjögren's syndrome B antibody (anti-SSB), and IgA, IgG, and IgM. No trial employed quality of life to evaluate clinical outcome. Adverse events were reported in 36 trials.

Information on participants, interventions, comparators, and outcomes reported in each trial was presented in Table 1; and detailed data from the outcome report was shown in Table 2 .

3.3. Risk of Bias within Studies. Methods to generate allocation sequence were reported in 14 trials $[12,13,15,18$, 19, 23-25, 33, 39, 40, 47, 52, 56]. These included random number tables, computer statistical software, and block randomization. One trial reported allocation concealment by using sealed opaque envelopes [65]. Blinding was employed in five trials [24, 25, 33, 37, 65]. In Yu [24] and Lian [33]'s trial, placebo of CHM decoctions was used in the control group. In Zhang [25], Zhong [37], and Wang [65] trials, real CHM and placebo of conventional medicines were used in the treatment group, while real conventional medicine and placebo CHM were used in the control group. Placebos were indistinguishable from the real treatment with respect to color, smell, and packaging in these trials. Another seven trials reported participant drop out and loss to followup $[12,25,32,33,38,47,49]$. Base-line data of participants in all included trials was comparable with no other risk of bias detected.

\subsection{Effect Estimation}

3.4.1. Response Rate. The response rate was defined as numbers of participants in both treatment and control 


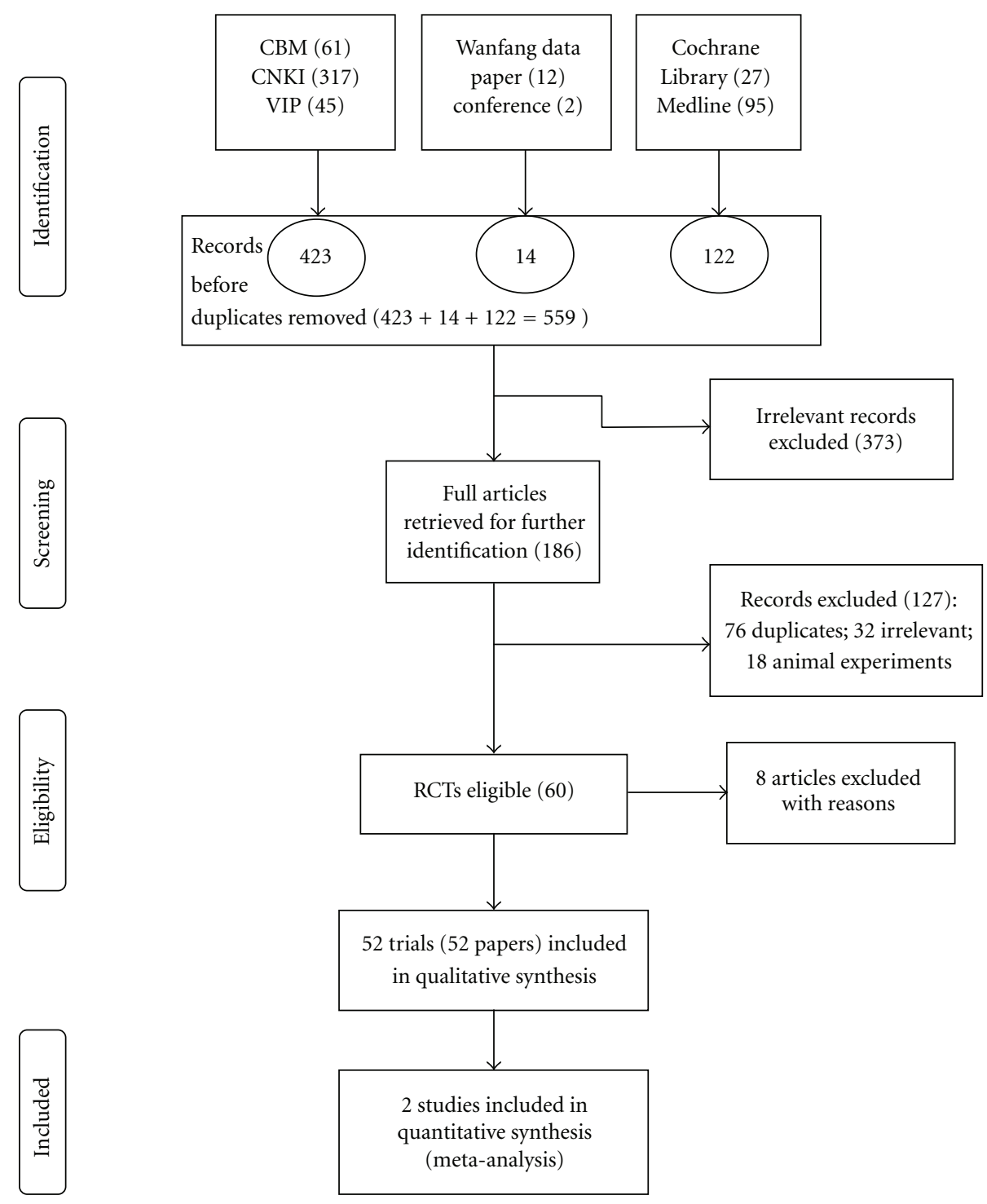

FIGURE 1: Flow-chart of study selection. PRISMA (preferred reporting items for systematic reviews and meta-analyses) flow-chart of study selection.

group who had global or partial symptomatic improvement, defined by either physician assessment or by laboratory tests. $94.2 \%(49 / 52)$ of the trials reported response rate but none of the trials reported the estimated effect using RR and 95\% CIs. According to the primary data reported in their papers, we calculated the RR and 95\% CIs of response rates, and the results showed that $65.3 \%(32 / 49)$ of the trials found a significant difference between CHM treatment and control groups. The overall estimates of effect within the four comparisons were:

CHM versus placebo $(n=2)$ : two trials compared a single herbal formula to placebo and only one [33] found significant improvement (RR: $4.25,95 \%$ CI: 1.76 to 10.29 ; $P<0.001)$, the other [24] used a cross-over randomized design, and no difference was found (RR: 1.0, 95\% CI: 0.84 to $1.18 ; P=0.72)$.
CHM versus conventional medicine $(n=19)$ : all trials in this category reported the response rate and $63.2 \%(12 / 19)$ found significant differences favoring the CHM treatment over the control groups $[19,21,23,39,40,50,52,55,57$, 58, 63, 65] (Table 2). Conventional therapies included artificial tears, prednisone, methotrexate, cyclophosphamide, vitamin, and other symptomatic management.

CHM plus conventional medicine versus conventional medicine $(n=28)$ : 26 trials in this category reported the response rate, of which $69.2 \%(18 / 26)$ found significant differences favoring the CHM treatment over the control groups $[12,16,18,20,25-27,29-31,35,37,42-44,48$, $49,61]$. Pooled data of two trials [12, 25] found that Jinju Qingrun capsule plus eye drops was more effective than hydroxychloroquine sulfate plus eye drops (RR: 1.28, 95\% CI: 1.02 to $1.62 ; P=0.04)$. 
CHM plus acupuncture/acupressure versus conventional medicine $(n=3)$ : two trials in this category reported the response rate. One trial compared CHM plus acupuncture and conventional medicine with conventional medicine, and found a significant better effect from the combination therapy (RR: 1.96, 95\% CI: 1.01 to $3.81 ; P<0.05$ ) [64]. Another trial [54] compared CHM plus ear acupressure with conventional medicine but failed to find any significant difference (RR: $1.15,95 \% \mathrm{CI}: 0.93$ to $1.43 ; P=0.2$ ).

In the above 49 trials, only one trial was assessed as having a low risk of bias [65], reporting adequate method of allocation concealment, double-blinding methods, and no withdrawals during the study. This trial compared a CHM decoction (Radix Rehmanniae Recens, Liriope spicata, Leguminosae, Pseudostellaria heterophylla, Cornu Bubali, Radix Salviae Miltiorrhizae) with hydroxychloroquine in treating PSS. The results showed that this herbal decoction demonstrated a superior effect to hydroxychloroquine in the improvement of eye and mouth symptoms (RR: 1.66, 95\% CI: 1.08 to $2.56 ; P<0.05)$.

3.4.2. Lacrimal Function. A total of 20 trials employed the Schirmer test to determine whether the participants' eyes could produce normal tears to keep the eyes moist. Five out of seven found significant improvement favoring the CHM over the conventional medicine [32, 40, 45, 47, 58], and $83.3 \%(10 / 12)$ of trials found significant difference between the CHM plus conventional medicine, and the conventional medicine alone $[13,17,20,25,30,38,46,59-61]$. In addition, Yang [53] found that a herbal formula plus acupuncture was superior to methotrexate for improving lacrimal function (RR: 2.98, 95\% CI: 2.01 to $3.95 ; P<0.00001$ ).

3.4.3. Salivary Gland Function. There were 21 trials that either used a salivary flow rate test or sugar-melt test to determine the function of salivary glands, of which $52.4 \%$ $(11 / 21)$ trials found that there was a significant difference between treatment and control groups, which favored the CHM 2 trials compared CHM to conventional medicine $[32,50] ; 8$ trials compared CHM plus conventional medicine to conventional medicine alone $[12,13,25,46,49,59-$ 61]; and 1 trial used CHM plus acupuncture compared to conventional medicine [53]. The other trials failed to find a significant difference between treatment and control groups. One trial measured the salivary flow rate and the other tested sugar-melted time, and the data could not be converted and synthesized.

3.4.4. Quality of Life. No trials used validated quality of life (QoL) measures, such as WHOQOL or SF-36, to evaluate clinical effectiveness. Among the included trials, one applied self-developed criteria to evaluate QoL, but found no statistical difference between the treatment and control group [23].

3.5. Adverse Effects. Adverse effects were reported in 19 trials $[12,13,15,17,18,20,25,32,34,37-39,44,46$, $47,51,56,57,61]$, and another seven reported that no adverse effects occurred during the study $[14,23,24$,
31, 33, 49, 59]. The adverse effects reported in CHM treatment included nausea, abdominal pain, diarrhea, and other minor gastrointestinal symptoms. When conventional medicines were used, either as the sole intervention or in conjunction with CHM, adverse effects included vomiting, diarrhea, insomnia, rashes, blurred vision, edema, central obesity, increased ALT level, mild hepatic dysfunction, renal dysfunction, anemia, increased fasting blood glucose level, hypertension, hyperlipoidemia, and leucopenia occurred.

A funnel plot was not conducted due to clinical heterogeneity of the included trials.

\section{Discussion}

4.1. Analysis of Effectiveness and Safety. The data within this paper involving 52 trials cannot be synthesized into a meta-analysis because of their heterogeneity. The included trials report moderate effectiveness (overall response rate and function improvement on lacrimal and salivary gland) for PSS treatment for CHM when compared with conventional medicine or placebo. We evaluated the safety reports from the CHM: the adverse effects occurred in the CHM group appear less than those in the conventional medicine group.

There is no known cure in conventional medicine for PSS and this paper provides preliminary evidence that CHM may be a promising and safe intervention for this chronic longterm condition. However, there are important limitations in this paper that weaken the recommendation of $\mathrm{CHM}$ for their clinical use.

\subsection{Limitations of the Systematic Review}

4.2.1. Methodological Quality of Included Trials. Almost all the randomized trials of CHM identified in this review evaluating treatment for PSS have a high risk of bias. Only $28.8 \%(15 / 52)$ of the included trials reported the randomization process and few trials reported allocation concealment or blinding, and no trial reported intention-totreat analysis.

However, it is important to note that the methodological quality of RCTs in this field has shown signs of improvement over the last 3 years. Reports on the generation of allocation sequence account for $13.0 \%$ (3/23) of included trials published between 1997 and 2007, compared to 25.0\% (4/16) in RCTs published between 2008 and 2009, and 61.5\% (8/13) in RCTs published between 2010 and 2011. Application of blinding in study design accounted for $0 \%(0 / 23)$ of included trials published between 1997 and 2007, compared to $17.2 \%$ (5/29) that were published between 2008 and 2011. Reports of dropout rates and those lost to followup accounted for $8.7 \%(2 / 23)$ of included trials published between 2008 and 2011 , compared to $17.2 \%$ (5/29) of trials published between 2008 and 2011. Nevertheless, the current quality of RCTs' in CHM is still unsatisfactory, because only one in 52 trials reported all the items in the Cochrane Risk of Bias tool adequately, while all other trials were of moderate or high risk of bias. Since most of the trials report positive results, and no difference could be found between trials published 


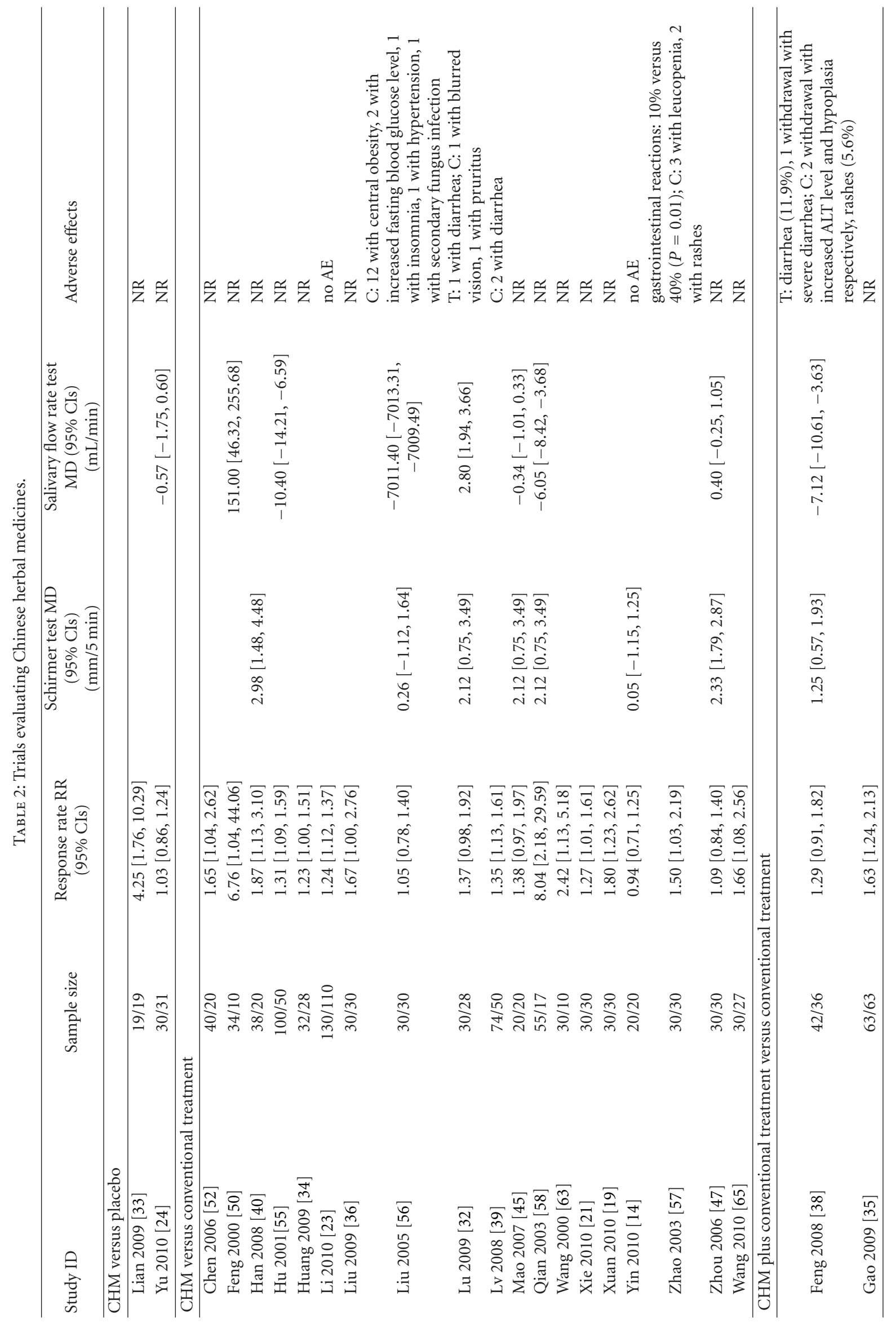



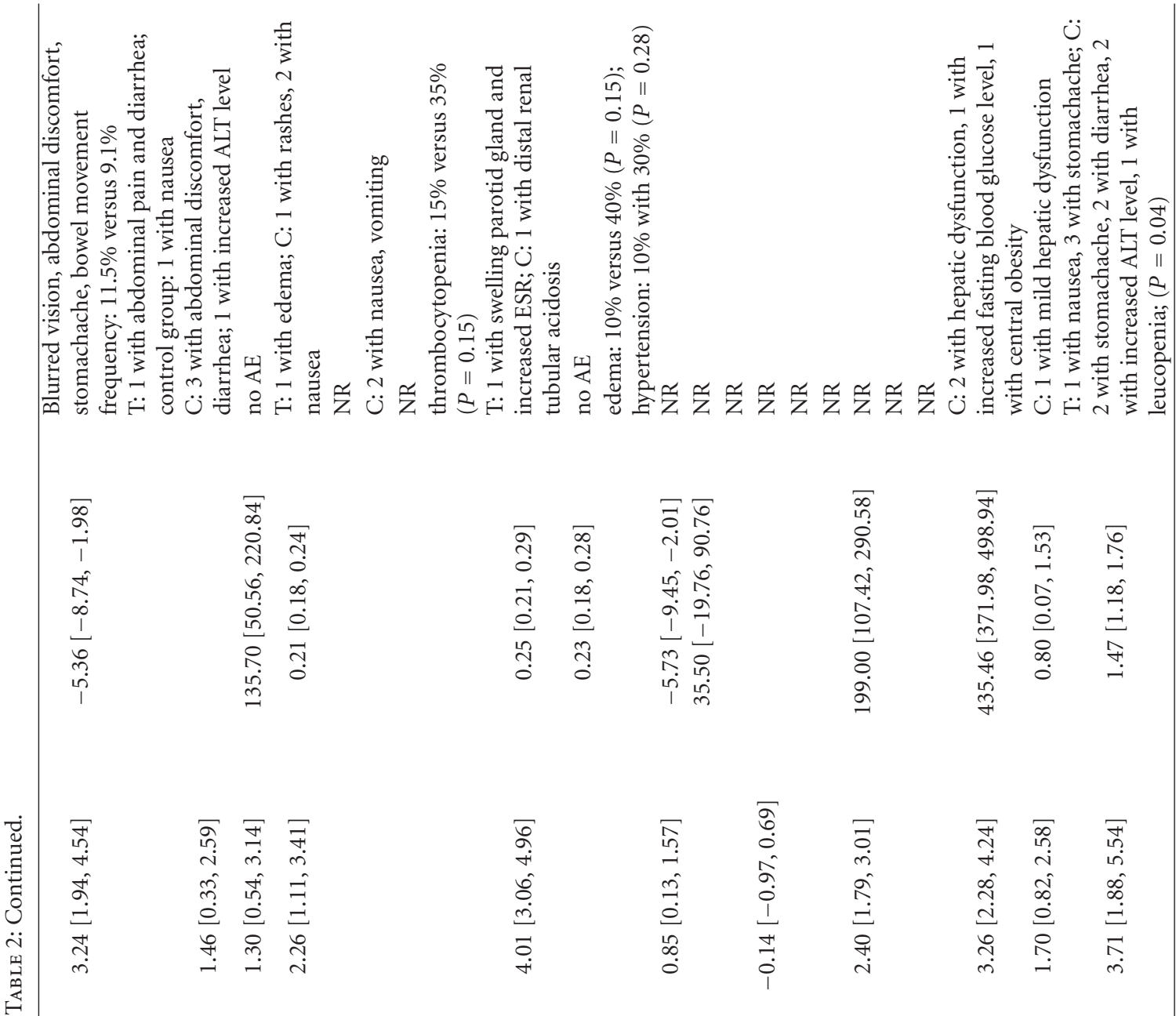

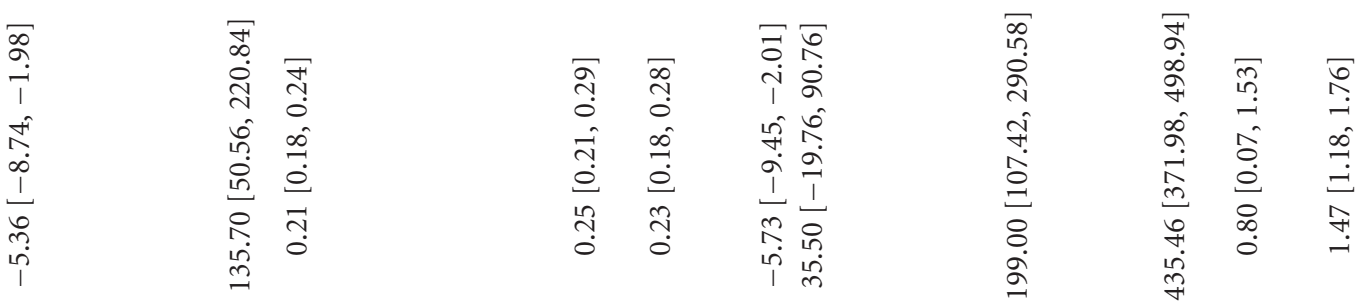

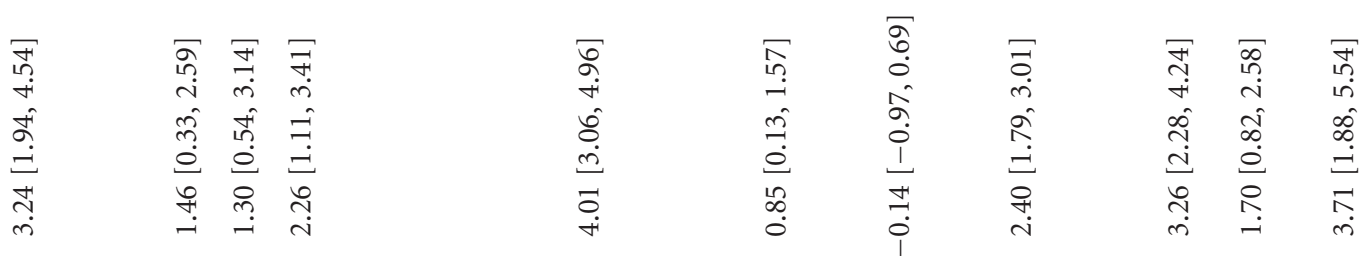

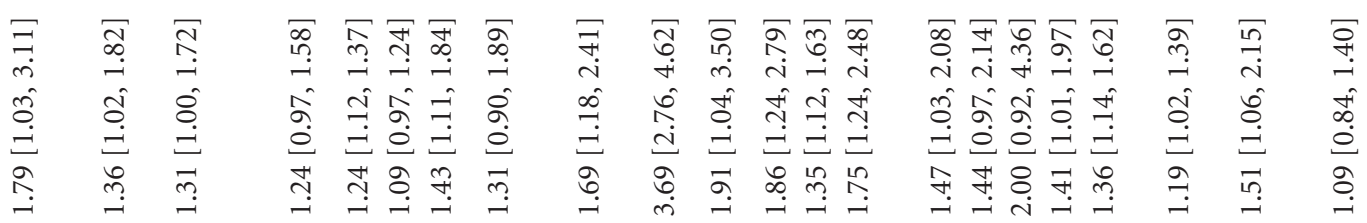

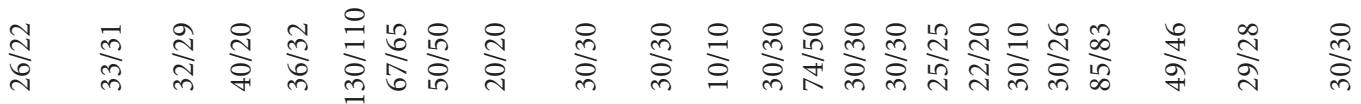

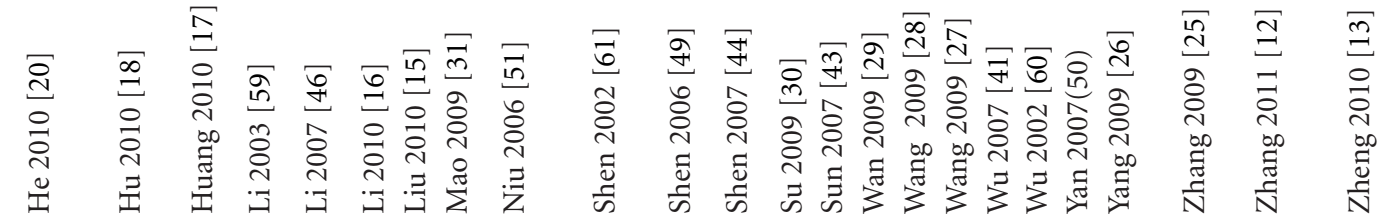




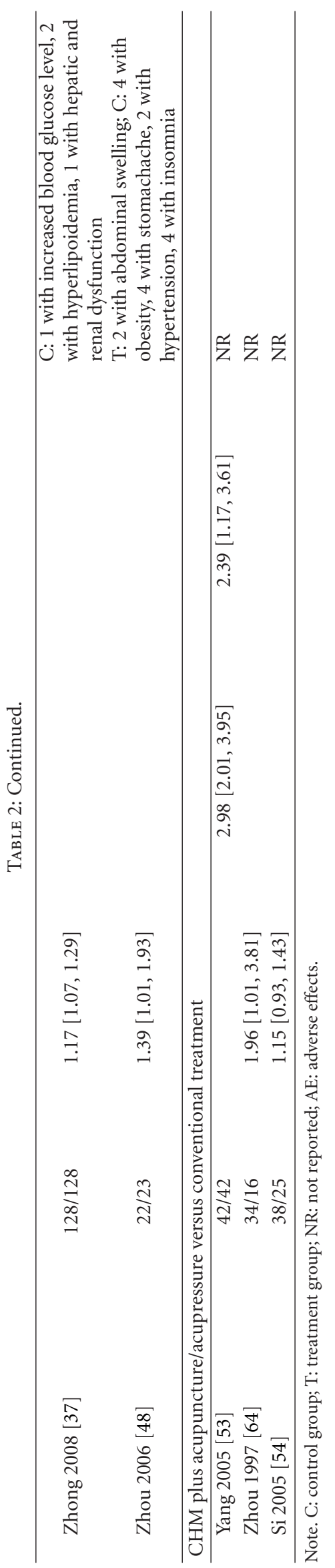


TABLE 3: Search strategy.

\begin{tabular}{|c|c|}
\hline Database & Search strategy \\
\hline PubMed & $\begin{array}{l}\text { "Sjögren's syndrome" (mesh) and ("humans" (MeSH terms) and } \\
\text { (meta-analysis (ptyp) OR randomized controlled trial (ptyp)) }\end{array}$ \\
\hline Cochrane Library & $\begin{array}{l}\text { "Primary sjögren's syndrome in record title in cochrane central register of } \\
\text { controlled trials" }\end{array}$ \\
\hline China Knowledge Resource Integrated Database (CNKI) & "Sjögren's syndrome in record title AND random* in all text" \\
\hline VIP Database for Chinese Technical Periodicals (VIP) & "Sjögren's syndrome in record title AND random* in all text" \\
\hline Wanfang Data & "Sjögren's syndrome in record title AND random* in all text" \\
\hline Chinese Biomedical Database (CBM) & "Sjögren's syndrome in record title AND random* in all text" \\
\hline
\end{tabular}

prior to 2008 and those with better quality studies published from 2008 to 2011 (see Table 2), we consider that this may suggest therapeutic benefit of CHM.

4.2.2. Inconsistency and Heterogeneity of Response Rates. The majority $(94.2 \%, 49 / 52)$ of included trials used response rate (total effectiveness rate) as the primary outcome measure. This is a composite outcome index, integrating factors from symptoms, signs, and laboratory findings. This subjective and vague outcome index is prone to bias and misinterpretation, particularly if there is insecure or no blinding. In the included trials, the procedure for determining effectiveness rate has not been standardized and different approaches to defining the response rate have led to an inconsistency and heterogeneity in the assessment of the efficacy of these interventions. Under these circumstances, although the results of most trials suggest CHM can improve "response rate" more significantly than standard conventional medicine or placebo, we must still remain highly circumspect about the specific effect of CHM on PSS.

4.2.3. Complexity of Interventions. In accordance with TCM theory interventions were designed to be adapted according to the specific presentations of PSS, so they varied from trial to trial. In addition CHM was frequently used in conjunction with other treatments including conventional herbal medicine, placebo, and acupuncture. This complexity of intervention makes it difficult to use double blinding (patients and physicians). It also complicates an assessment of the effects of different components of the interventions. In these instances we can only report the effectiveness of a whole therapeutic system such as TCM rather than a single isolated treatment.

4.3. Other Limitations of Included Trials. Diagnostic criteria: diagnosis of PSS was not consistent across included trials. About $63 \%$ of the trials employed international criteria for diagnosis of Sjögren's syndrome, and the remaining trials cited domestic criteria or unestablished criteria. This may increase variations among participants.

4.3.1. Treatment Duration and Follow-Up. PSS is a systemic autoimmune disease and requires long-term care and treatment. Therefore, evaluation of the effect of an intervention should be based on long-term treatment and followup.
However in the included 52 trials, only six trials continued follow up beyond the six-month treatment $[19,30,38,52$, $55,61]$, and a further four trials had a relatively short 3-6months followup [38, 44, 49, 61]. This is insufficient for the assessment of the long term effectiveness of CHM.

4.3.2. Quality of Life. fatigue is a common symptom in patients with Sjögren's syndrome, and greatly influences patients' quality of life $[80,81]$. The SF-36 has been widely used in this context $[2,81]$ but none of the Chinese studies employed a validated instrument for quality of life evaluation for PSS.

4.4. Recommendations for Further Research. Studies are beginning to, and must continue to, report randomization sequence generation and allocation concealment in detail and employ blinding in outcome measurement and evaluation as well as an intention to treat analysis and a clear description of dropout. If we want to study the specific efficacy of CHM interventions, double-blind and placebo controlled trials are necessary. However, due to the limited evidence available for conventional drugs most frequently used in PSS [10], it is reasonable to justify employing pragmatic clinical trials which either add CHM to conventional medicines or use CHM in a comparative effectiveness research versus conventional medicine rather than comparing CHM with placebo.

The Medical Research Council (MRC) guidelines for complex interventions highlighted that complex interventions might work best if tailored to local circumstances rather than being completely standardized [82]. Since the interventions were designed to adapt TCM syndrome differentiations to the specific characteristics of individual PSS presentations, and complex treatment "packages" were employed in these trials (including CHM, acupuncture as well as integrating CHM with conventional medicine), we suggest that a whole system approach should be adopted. This would involve using the various treatment modalities within TCM (a combination of CHM, acupuncture, and dietary and lifestyle advice) and comparing that with a package of conventional medicine treatment.

We recommend that the diagnostic criteria from the 2002 International Classification of Sjögren's syndrome be used for future TCM PSS research. We also recommend an extended course of treatment and followup time, using 
both quantitative and qualitative outcomes to make a proper assessment of the effectiveness of this intervention. Invalidated outcomes such as "response rate" are unreliable, difficult to standardize and interpret, and should not be used.

\section{Conclusions}

52 RCTs were analyzed in this systematic review, testing various CHMs in the treatment of PSS. The findings from these trials suggest that CHM delivered either as a sole treatment or in conjunction with conventional medicine may be more effective than conventional medicine in managing PSS symptoms-with specific reference to lacrimal and salivary gland function. However, a high risk of bias in these studies and the heterogeneity of the CHM intervention and outcome assessment suggest that these positive findings must be interpreted with considerable caution; we cannot recommend any specific Chinese herbal medicines for clinical use. This preliminary evidence supports the continuing use and evaluation of individualized CHM as a potentially promising and safe intervention for this syndrome. We recommend that adequately powered and rigorously conducted further research should employ a variety of trial methodologies including double-blind placebo-controlled RCTs, pragmatic, and comparative equivalence trials, to investigate $\mathrm{CHM}$ treatment for PSS. If the evidence justifies it CHM can then be more widely recommended as a treatment for this common and troublesome condition.

\section{Conflict of Interests}

The authors declared no conflict of Interests.

\section{Acknowlegments}

The study was supported by a grant from international cooperation project (no. 2009DFA31460) from the Ministry of Science and Technology of China, and in part supported by the basic operational funding for scientific research from Beijing University of Chinese Medicine (2011-CXTD-09). The funders had no role in study design, data collection and analysis, decision to publish, or preparation of the paper.

\section{References}

[1] Chinese Medical Association of Rheumatology, "Guidelines for the diagnosis and treatment of Sjogren's syndrome (draft)," Chinese Journal of Rheumatology, vol. 7, pp. 446-448, 2003 (Chinese).

[2] J. M. Meijer, P. M. Meiners, J. J. R. H. Slater et al., "Healthrelated quality of life, employment and disability in patients with sjögren's syndrome," Rheumatology, vol. 48, no. 9, pp. 1077-1082, 2009.

[3] M. Ramos-Casals, A. G. Tzioufas, and J. Font, "Primary Sjögren's syndrome: new clinical and therapeutic concepts," Annals of the Rheumatic Diseases, vol. 64, no. 3, pp. 347-354, 2005.

[4] C. G. Helmick, D. T. Felson, R. C. Lawrence et al., "Estimates of the prevalence of arthritis and other rheumatic conditions in the United States. Part I," Arthritis and Rheumatism, vol. 58, no. 1, pp. 15-25, 2008.

[5] E. Thomas, E. M. Hay, A. Hajeer, and A. J. Silman, "Sjogren's syndrome: a community-based study of prevalence and impact," British Journal of Rheumatology, vol. 37, no. 10, pp. 1069-1076, 1998.

[6] Y. Alamanos, N. Tsifetaki, P. V. Voulgari, A. I. Venetsanopoulou, C. Siozos, and A. A. Drosos, "Epidemiology of primary Sjögren's syndrome in north-west Greece, 19822003," Rheumatology, vol. 45, no. 2, pp. 187-191, 2006.

[7] M. Tomšič, D. Logar, M. Grmek, T. Perkovič, and T. Kveder, "Prevalence of Sjogren's syndrome in Slovenia," Rheumatology, vol. 38, no. 2, pp. 164-170, 1999.

[8] N. Z. Zhang, C. S. Shi, Q. P. Yao et al., "Prevalence of primary Sjogren's syndrome in China," Journal of Rheumatology, vol. 22, no. 4, pp. 659-661, 1995.

[9] M. Ramos-Casals and J. Font, "Primary Sjögren syndrome," in Current Diagnosis and Treatment in Rheumatology, J. Imboden, D. Hellmann, and J. Stone, Eds., pp. 237-245, McGraw-Hill, New York, NY, USA, 2007.

[10] M. Ramos-Casals, A. G. Tzioufas, J. H. Stone, A. Sisó, and X. Bosch, "Treatment of primary Sjögren syndrome: a systematic review," Journal of the American Medical Association, vol. 304, no. 4, pp. 452-460, 2010.

[11] H. Luo, M. Han, and J. P. Liu, "Systematic review and metaanalysis of randomized controlled trials of Chinese herbal medicine in the treatment of Sjögren' s syndrome," Journal of Chinese Integrative Medicine, vol. 9, no. 3, pp. 257-274, 2011 (Chinese).

[12] S. Y. Zhang, G. Guo, J. Liang, H. Y. Li, H. S. Zhang, and H. X. H. Zhu, "Clinical therapeutic effect of self-made Jinjuqingrun capsule on 30 patients with primary Sjögren's syndrome," Chinese Journal of Difficult and Complicated Cases, vol. 10, pp. 835-838, 2011 (Chinese).

[13] H. X. Zheng, X. J. Tao, and J. N. Yu, "Clinical observation on Runzao formula in treating 30 cases of primary Sjögren's syndrome," Chinese Journal of Integrated Traditional and Western Medicine, vol. 30, pp. 436-438, 2010 (Chinese).

[14] G. F. Yin, M. Yue, F. H. Liu, and J. P. Nie, "Yangyin Qingre Jiedu decoction in treating 20 cases of Sjögren's syndrome," Traditional Chinese Medicinal Research, vol. 23, pp. 40-42, 2010 (Chinese).

[15] H. W. Liu and X. Wang, "Yuquan pill in combination with Shengmai injection in the treatment of 67 cases of Sjögren's syndrome," Journal of China Traditional Chinese Medicine Information, vol. 2, p. 72, 2010 (Chinese).

[16] J. Li, C. Q. Si, and Q. Li, "Clinical observation on Qingre Quyu decoction in the treatment of primary Sjögren's syndrome," The Journal of Medical Theory and Practice, vol. 23, p. 561, 2010 (Chinese).

[17] X. J. Huang, G. H. Xu, L. M. Li, and E. C. Cui, "Clinical observation of Shenmai injection in treatment of primary Sjögren syndrome," China Pharmacy, vol. 21, pp. 724-725, 2010 (Chinese).

[18] J. G. Hu, X. J. Chen, J. H. Gu, and J. C. Mao, "Therapeutic effect of Ziyin Yangxue Qingre formula on Sjögren's syndrome: a clinical observation on 33 cases," Journal of Traditional Chinese Medicine, vol. 51, pp. 226-228, 2010 (Chinese).

[19] J. Xuan and P. A. Shen, "Clinical research on the effect of Shenglu Runzao decoction on treating Sjögren's syndrome," Chinese Journal of Practical Stomatology, vol. 3, pp. 739-740, 2010 (Chinese).

[20] H. He, "Clinical observation of hydroxychloroquine and total glycosides of paeony for the treatment of Sjögren's syndrome," 
Hubei Journal of Traditional Chinese Medicine, vol. 32, pp. 3031, 2010 (Chinese).

[21] W. J. Xie, S. P. Jiang, S. J. Xie, and N. Li, "Clinical research of yangyin decoction for the treatment of Sjögren's syndrome," Chinese Journal of Ethnomedicine and Ethnopharmacy, vol. 19, pp. 173-174, 2010 (Chinese).

[22] Y. Dong, Y. Zhao, and X. Guo, "Preliminary diagnostic criteria for primary Sjogren's syndrome in China," Chinese Journal of Internal Medicine, vol. 35, no. 2, pp. 114-117, 1996 (Chinese).

[23] S. Y. Li, The Theoretical Studies and Clinical Research on Sjögren's Syndrome from Spleen-Yin Deficiency, Nanjing University of Chinese Medicine, Nanjing, China, 2010.

[24] Z. M. Yu, Clinical Research of Professor Luzhizheng's Nourishing Qi and Yin Treatment Method for Dry-Mouth of Primary Sjögren's Syndrome, Beijing University of Chinese Medicine, Beijing, China, 2010.

[25] S. Y. Zhang, R. Li, X. H. Zhu, S. Y. Zhang, Z. C. Wu, and F. B. Jiang, "Clinical observation on the effect of Jinju Qingrun capsule on 50 cases of primary Sjögren's syndrome," Journal of Traditional Chinese Medicine, vol. 50, pp. 708-711, 2009 (Chinese).

[26] A. Q. Yang, "Clinical observation of integrated traditional Chinese and western medicine in treating 85 cases of Sjögren's syndrome," China Medical Herald, vol. 6, pp. 77-78, 2009 (Chinese).

[27] Z. Z. Wang, "Clinical study on patient with primary Sjögren's syndrome with traditional Chinese medicine and western medicine," Chinese Journal of Modern Drug Application, vol. 16, pp. 46-47, 2009 (Chinese).

[28] W. P. Wang and S. M. Shi, "Clinical observation on effectiveness of self-made Yangyin Jianpi Huoxue decoction in combination with conventional therapy in the treatment of Sjögren's syndrome," China Journal of Traditional Chinese Medicine and Pharmacy, vol. 24, pp. 902-903, 2009 (Chinese).

[29] Q. B. Wan, "Integrated traditional Chinese and western medicine in the treatment of Sjögren's syndrome," Hubei Journal of Traditional Chinese Medicine, vol. 31, pp. 26-27, 2009 (Chinese).

[30] X. Su and N. Zhang, "The clinical study of traditional Chinese medicine for Yangyin Huoxue Shengjin Fang in treating yin asthenia generating intrinsic heat and blood stasis due to loss of body fluid of Sjögren syndrome," Chinese Archives of Traditional Chinese Medicine, vol. 27, pp. 2264-2268, 2009 (Chinese)

[31] L. P. Mao, B. Han, P. S. Yan, A. L. Liu, and A. M. Shi, "Clinical observation of integrated traditional Chinese and western medicine in treating 50 cases of Sjögren's syndrome," Chinese Journal of Gerontology, vol. 29, pp. 2811-2812, 2009 (Chinese).

[32] Y. Lu and S. Jin, "Clinical study on Shengjin granule in the treatment of primary Sjögren's syndrome with yindeficiency and collateral stagnation syndrome," Journal of Nanjing University of Traditional Chinese Medicine, vol. 25, pp. 421-423, 2009 (Chinese).

[33] J. Lian, Clinic Research on Treating Primary Sjögren's Syndrome with the Method of Yangyin Shengjin, Runzao Tongluo Method, Nanjing University of Chinese Medicine, Nanjing, China, 2009.

[34] X. J. Huang, X. h. Tong, and Y. P. Zhang, "Clinical research on patients of primary Sjögren's syndrome treated by traditional Chinese medicine of Yiqi Yangyin Huoxue method and anethole trithione," Aerospace Medicine, vol. 20, pp. 65-66, 2009 (Chinese).

[35] L. Gao, L. H. Ma, and X. D. Ji, "Clinical observation of integrated traditional Chinese and western medicine in the treatment of Sjögren's syndrome," Chinese Medicine Modern Distance Education of China, vol. 7, no. 3, p. 30, 2009 (Chinese).

[36] F. Liu, Nourish Yin and Tonify Kidney Moisten Dryness and Remove Blood Stasis Primary Sjogren's Syndrome the Clinical Treatment, Chengdu University of Chinese Medicine, Chengdu, China, 2009.

[37] Q. C. Zhong, "Clinical research on Yangyin Tongluo method in treating Sjögren's syndrome," in Basic and Clinical Research on Collateral Disease Theory, Y. L. Wu, Ed., vol. 4, pp. 387-390, Military Medical Science, Beijing, China, 2008.

[38] F. H. Feng and E. Z. Zhang, "Total glycosides of paeony in the treatment of 42 cases of Sjögren's syndrome," Traditional Chinese Medicinal Research, vol. 21, pp. 35-37, 2008 (Chinese).

[39] W. Z. Lv, C. X. Li, G. J. Sun, Y. F. Wang, and Z. H. Liu, "Clinical research on Jinyuan decoction for 74 cases of primary Sjögren's syndrome," Journal of Traditional Chinese Medicine, vol. 49, pp. 29-31, 2008 (Chinese).

[40] S. H. Han, S. Jin, Z. T. Liu, G. Wei, J. Wu, and B. B. Zhu, "Clinical study on 38 cases of primary Sjogren's syndrome patients treated with Xuanfei Bujin particles," Chinese Journal of Information on Traditional Chinese Medicine, vol. 15, pp. 1416, 2008 (Chinese).

[41] C. H. Wu and H. T. Wang, "Clinical observation on modified Maiwei Dihuang decoction in the treatment of Sjögren's syndrome," Beijing Journal of Traditional Chinese Medicine, vol. 26, pp. 36-37, 2007 (Chinese).

[42] L. P. Yan, "Integrated traditional Chinese and western medicine in the treatment of 30 cases of Sjögren's syndrome," Journal of Changchun University of Traditional Chinese Medicine, vol. 23, p. 80, 2007 (Chinese).

[43] G. J. Sun, C. X. Li, and W. Z. Lv, "Clearing and dredging therapy in the treatment of 74 cases of primary Sjögren's syndrome," Shandong Journal of Traditional Chinese Medicine, vol. 26, pp. 456-458, 2007 (Chinese).

[44] Y. B. Shen and C. M. Zheng, "Clinical observation on the effectiveness of compound glycyrrhizin in the treatment of primary Sjögren's syndrome," China Prescription Drug, vol. 9, p. 76, 2007 (Chinese).

[45] J. C. Mao, X. J. Chen, L. Su, J. H. Gu, and X. Y. Chen, "Clinical effects of Yi-qi-jian-pi therapy on Sjögren's syndrome," Chinese Journal of Clinical Pharmacy, vol. 16, pp. 231-233, 2007 (Chinese).

[46] F. Li, J. H. Yao, F. X. Zhang, L. J. Sun, J. M. Tao, and X. R. Ning, "Clinical observation on treatment of primary Sjögren's syndrome by compound glycyrrhizin injection," China Pharmacy, vol. 18, pp. 858-859, 2007 (Chinese).

[47] S. G. Zhou, "Dryness-clearing, detoxifying, yin-tonifying and collateral vessel-freeing therapy in the treatment of 30 cases of Sjögren's syndrome," Chinese Journal of Information on Traditional Chinese Medicine, vol. 13, pp. 66-67, 2006 (Chinese).

[48] D. H. Zhou, Y. Xu, J. Wu, Y. Jin, Q. D. Zhang, and Z. Q. Zhou, "Clinical observation on Dandi Qiongyu granules in the treatment of primary Sjögren's syndrome," Journal of Emergency in Traditional Chinese Medicine, vol. 15, pp. 252253, 2006 (Chinese).

[49] K. Shen, H. L. Ma, S. M. Guo, and L. K. Lv, "Clinical study of treating of primary Sjögren's syndrome by Runzao oral liquid," Hebei Medicine, vol. 12, pp. 124-126, 2006 (Chinese).

[50] Y. H. Feng, Z. Q. Tian, D. B. Song, Y. F. Zhou, and J. Zhang, "Observation on the effectiveness of Shengjin Runzao granules in the treatment of primary Sjögren's syndrome," Lishizhen 
Medicine and Materia Medica Research, vol. 11, p. 327, 2000 (Chinese).

[51] Y. F. Niu, J. Liu, C. B. Huang, and X. Chen, "The clinical effect of invigorating spleen to eliminate dampness and cooling heat in treating SS," Chinese Journal of Clinical Healthcare, vol. 9, pp. 445-447, 2006 (Chinese).

[52] X. Y. Chen, X. J. Chen, and J. H. Gu, "Suangan Shengjin therapy in the treatment of 40 cases of Sjögren's syndrome," Shaanxi Journal of Traditional Chinese Medicine, vol. 27, pp. 1361-1363, 2006 (Chinese).

[53] X. Y. Yang and Y. Wang, "Clinical observation on acupuncture in combination with herbal medicine in the treatment of 42 cases of Sjögren's syndrome," Journal of Shandong University of Traditional Chinese Medicine, vol. 29, pp. 292-293, 2005 (Chinese).

[54] X. W. Si, "Clinical observation on herbal medicine in combination with pressing ear in the treatment of 38 cases of Sjögren's syndrome," Jiangsu Journal Of Traditional Chinese Medicine, vol. 26, pp. 39-40, 2005 (Chinese).

[55] C. M. Hu, "Analysis of the therapeutic effect of Zengye mixture on 100 cases of Sjögren's syndrome," China Tropical Medicine, vol. 1, pp. 336-337, 2001 (Chinese).

[56] W. Liu, H. Wang, X. Y. Yang, F. Zuo, and F. Y. Chen, "Clinical observation on Qingzao formula in the treatment of Sjögren's syndrome," Chinese Journal of Integrated Traditional and Western Medicine, vol. 25, p. 53, 2005 (Chinese).

[57] Z. W. Zhao and W. R. Wang, "Modified Qiju Dihuang decoction in the treatment of 30 cases of primary Sjögren's syndrome," Chinese Journal of Traditional Medical Science and Technology, vol. 10, pp. 314-315, 2003 (Chinese).

[58] X. Qian, Y. Z. Ma, M. J. Zhang, W. Ji, S. J. Lu, and Y. Lu, "The clinical study of treatment for SS with Jinxueyuan," Chinese Journal of the Practical Chinese With Modern Medicine, vol. 16, pp. 975-977, 2003 (Chinese).

[59] C. X. Li, G. L. Yang, Y. H. Feng, Y. J. Shi, and Q. J. Tan, "Observation on effectiveness of Shengmai injection in the treatment of primary Sjögren's syndrome," Clinical Medicine of China, vol. 19, p. 807, 2003 (Chinese).

[60] S. F. Wu, "Ziyin Huoxue formula in the treatment of 30 cases of Sjögren's syndrome," Chinese Journal of Information on Traditional Chinese Medicine, vol. 9, pp. 51-52, 2002 (Chinese).

[61] K. Shen, "Liuwei Dihuang decoction in combination with Zengye decoction in the treatment of 30 cases of primary Sjögren's syndrome," Shandong Journal of Traditional Chinese Medicine, vol. 21, pp. 467-469, 2002 (Chinese).

[62] R. Manthorpe, K. Frost-Larsen, H. Isager, and J. U. Prause, "Sjogren's syndrome. A review with emphasis on immunological features," Allergy, vol. 36, no. 3, pp. 139-153, 1981.

[63] H. Wang, W. Liu, and F. Zuo, "Observation on the effectiveness of Runzao mixture in the treatment of 40 cases with Sjögren's syndrome," Journal of Tianjin College of Traditional Chinese Medicine, vol. 19, p. 29, 2000 (Chinese).

[64] Q. Zhou and H. Q. Zhu, "Integrated traditional Chinese and western medicine in the treatment of 34 cases of Sjögren's syndrome," Journal of New Chinese Medicine, vol. 29, pp. 2930, 1997 (Chinese).

[65] X. C. Wang, J. Y. Huang, Z. J. Xie, C. P. Wen, L. Y. Cao, and Y. S. Fan, "Influence of medicine of notifying qi to nourish yin and remove stasis on sexual hormone of dry syndrome," Journal of Zhejiang College of Traditional Chinese Medicine, vol. 33, pp. 48-50, 2010 (Chinese).
[66] C. Vitali, S. Bombardieri, R. Jonsson et al., "Classification criteria for Sjögren's syndrome: a revised version of the European criteria proposed by the American-European Consensus Group," Annals of the Rheumatic Diseases, vol. 61, no. 6, pp. 554-558, 2002.

[67] C. Vitali, S. Bombardieri, H. M. Moutsopoulos et al., "Preliminary criteria for the classification of Sjogren's syndrome: results of a prospective concerted action supported by the European community," Arthritis and Rheumatism, vol. 36, no. 3, pp. 340-347, 1993.

[68] J. P. T. Higgins and S. Green, The Cochrane Collaboration. Cochrane Handbook for Systematic Reviews of Interventions. Version 5.0.2., 2009, http://www.cochrane-handbook.org/ .

[69] J. N. T. Gu and Y. Ba, "Chinese integrative medicine for Sjögren's syndrome with blood system damage," Chinese Journal of Experimental and Traditional Medical Formulae, vol. 16, pp. 223-224, 2010 (Chinese).

[70] M. A. Zhou, H. Zhou, B. H. Zhuang, and Z. S. Zuo, "Clinical observation of Yangyin huoxue yishen decoction on treatment of primary Sjögren's syndrome with kidney damage," China Modern Medicine, vol. 17, pp. 5-6, 2010 (Chinese).

[71] H. Qin, "Combination of Yunvj and Yiwei decoction on the treatment of 25 cases of primary Sjögren's syndrome with spleen and stomach yin deficiency," Journal of Guangxi Traditional Chinese Medical University, vol. 13, pp. 13-15, 2010 (Chinese).

[72] Y. L. Zhu, N. Wei, and X. J. Hou, "Treatment of Sjögren's syndrome with Huoxuejiedu Formula in 63 cases," Journal of Beijing University of Traditional Chinese Medicine, vol. 17, pp. 1-3, 2010 (Chinese).

[73] Q. C. Zhong, Q. C. Zhang, Z. L. Wang, F. B. Jiang, X. Zhang, and X. H. Zhu, "Clinical observation on Sjögren's syndrome treated by runzaoling capsule combined with intraparotid injection," Chinese Journal of Information on TCM, vol. 13, pp. 24-27, 2006 (Chinese).

[74] X. R. Li, Curative Effect Observation of the Method of Moistening Dryness and Promoting Blood Circulation on Treating Sjögren's Syndrome and the Study of Its Effect on Salivary Electrolytes, Nanjing University of Chinese Medicine, Nanjing, China, 2010 (Chinese).

[75] H. D. Zhang, Y. J. Bian, Z. M. Yu, J. Lu, X. P. Tang, and Q. Jiang, "The influence to dry-mouth symptom and immue indexs of primary Sjögren's syndrome patients by using Lushi qingzao decoction," Shandong Journal of Traditional Chinese Medicine, vol. 29, pp. 815-817, 2010 (Chinese).

[76] C. J. Chen, J. H. Gu, and J. C. Mao, "Clinical research of Suangan Shenjin Formula for the treatment of Sjogren's syndrome with two kind of TCM differentiation syndrome," Traditional Chinese Medicine Journal of Rheumatology, vol. 9, pp. 12-15, 2006 (Chinese).

[77] N. Z. Zhang, Clinical Rheumatology, Shanghai Scientific and Technical Publishers, Shanghai, China, 1999 (Chinese).

[78] State administration of TCM China, Standard of the Diagnosis and Treatment Effect for Traditional Chinese Medicine Diseases, Nanjing University Press, Nanjing, 1994 (Chinese).

[79] X. Y. Zheng, Guidelines for Clinical Research on Chinese New Herbal Medicines (trial implementation), China Medical Science Press, Beijing, China, 2002 (Chinese).

[80] R. I. Fox, "Sjögren's syndrome," The Lancet, vol. 366, no. 9482, pp. 321-331, 2005.

[81] S. J. Bowman, D. A. Booth, R. G. Platts et al., "Measurement of fatigue and discomfort in primary Sjögren's syndrome using a new questionnaire tool," Rheumatology, vol. 43, no. 6, pp. 758 764, 2004. 
[82] P. Craig, P. Dieppe, S. Macintyre, S. Michie, I. Nazareth, and M. Petticrew, "Developing and evaluating complex interventions: the new Medical Research Council guidance," British Medical Journal, vol. 337, p. a1655, 2008. 


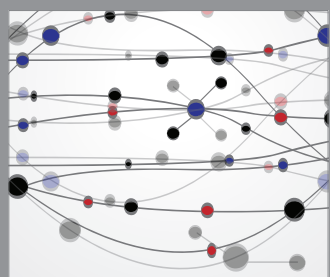

The Scientific World Journal
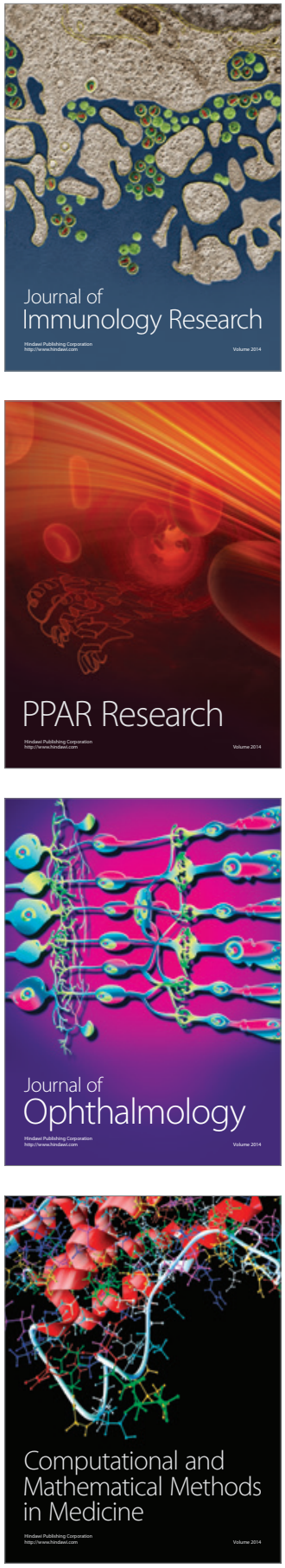

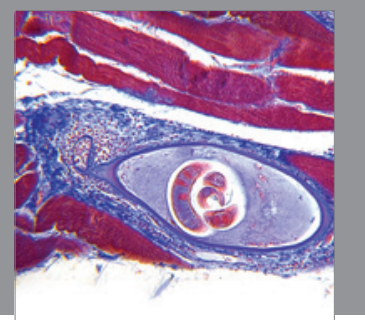

Gastroenterology

Research and Practice
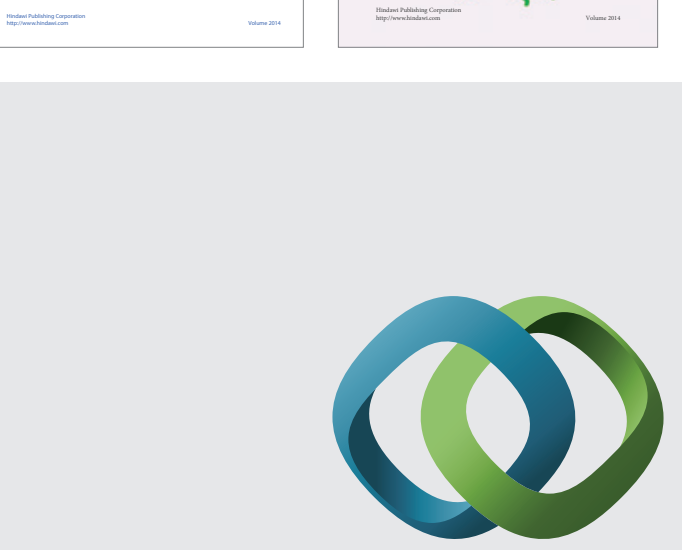

\section{Hindawi}

Submit your manuscripts at

http://www.hindawi.com
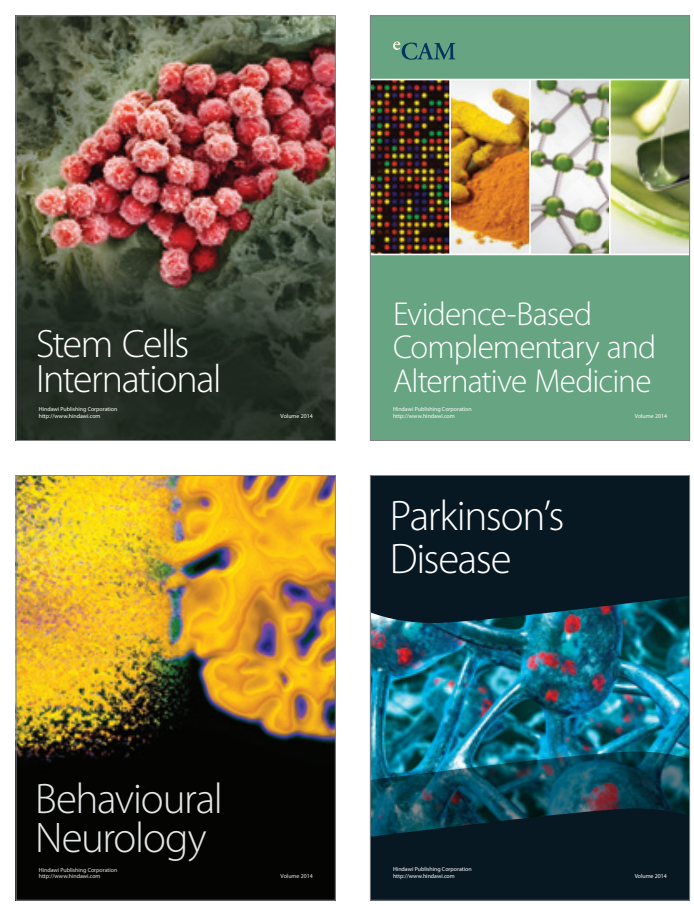

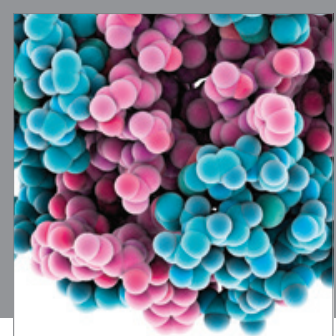

Journal of
Diabetes Research

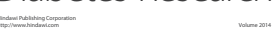

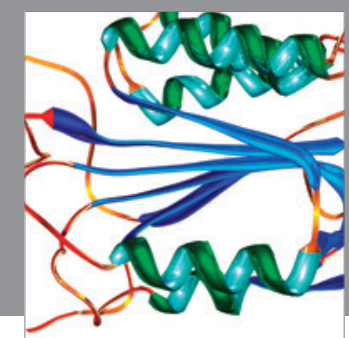

Disease Markers
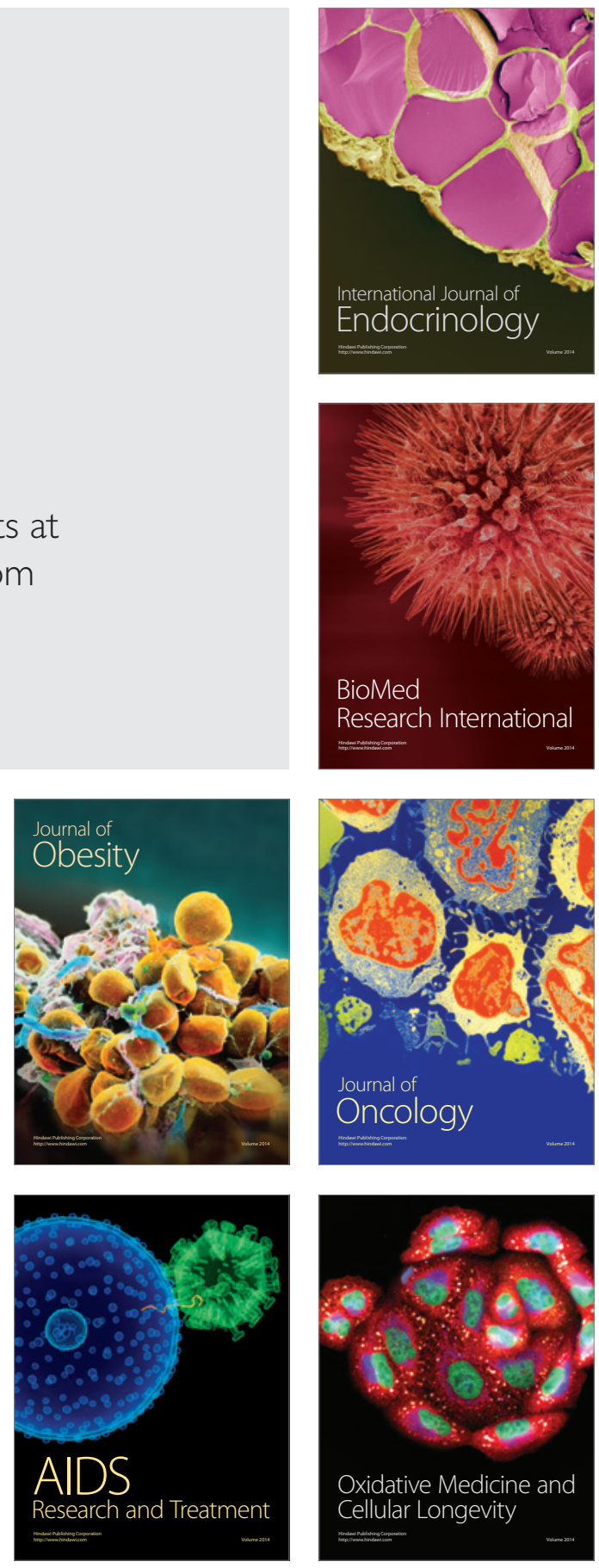